\title{
Construção e validação de conteúdo de cartilha educativa para prevenção de quedas no hospital
}

Construction and validation of educational booklet content for fall prevention in hospitals Elaboración y validación de contenido de cartilla educativa para prevención de caídas en el hospital

Maria Aline Moreira Ximenes' Natália Ângela Oliveira Fontenele ${ }^{1}$

Ismael Brioso Bastos 1

Thamires Sales Macêdo'

Nelson Miguel Galindo Neto ${ }^{2}$

Joselany Áfio Caetano ${ }^{3}$

Livia Moreira Barros ${ }^{1}$

\section{Descritores}

Educação em saúde; Acidentes por quedas: Prevenção de acidentes; Estudos de validação

Keywords

Health education; Accidental falls; Accident prevention; Validation studies

\section{Descriptores}

Educación en salud; Accidentes por caídas: Prevención de accidentes; Estudios de validación

Submetido 7 de Dezembro de 2018

Aceito

8 de Maio de 2019

\section{Resumo}

Objetivo: Construir e validar cartilha educativa para prevenção de quedas no hospital.

Métodos: Estudo metodológico desenvolvido a partir da construção, validação e avaliação da cartilha por 22 enfermeiros e 22 pacientes internados em clínica médica. Para a validação com juízes, avaliou-se objetivo, estrutura/apresentação e relevância do material e, na avaliação com pacientes, aplicou-se o instrumento Suitability Assesment of Materials (SAM). Utilizou-se o Índice de Validade de Conteúdo (IVC) para análise de cada etapa da validação, considerando válido os iguais ou superiores a 0,80.

Resultados: A cartilha intitulada "Cuidados para não cair nessa" é composta por 20 páginas. Na validação de conteúdo, os profissionais atribuíram o IVC global de 1,0 para objetivo, estrutura/apresentação e relevância do material. Na avaliação dos pacientes, o IVC global da cartilha foi de 0,98. Conclusão: A cartilha educativa é considerada válida quanto ao conteúdo e compreensível pelos pacientes de forma que possui utilização viável na educação em saúde para prevenção de quedas no hospital.

\section{Abstract}

Objective: To construct and validate an educational booklet for fall prevention in hospitals.

Methods: A methodological study developed from the construction, validation and assessment of the booklet by 22 nurses and 22 patients hospitalized in a medical clinic. For the validation with judges, objective, structure/presentation and relevance of the material were assessed and, in assessment with patients, the Suitability Assessment of Materials (SAM) tool. Content Validity Index (CVI) was used to analyze each validation step, considering valid values equal to or greater than 0.80 .

Results: The booklet titled "Careful not to fall for this" consists of 20 pages. In content validation, practitioners assigned the overall CVI of 1.0 for purpose, structure/presentation, and material relevance. In the assessment of patients, the overall CVI of the booklet was 0.98 .

Conclusion: The educational booklet is considered valid regarding the content and understandable by patients in a way that has a viable use in health education for fall prevention in hospitals.

\section{Resumen}

Objetivo: elaborar y validar cartilla educativa para prevención de caídas en el hospital.

Métodos: estudio metodológico desarrollado a partir de la elaboración validación y evaluación de la cartilla por 22 enfermeros y 22 pacientes internados en clínica médica. Para la validación con jueces, se analizó el objetivo, estructura/presentación y relevancia del material; y para la evaluación con pacientes, se aplicó el instrumento Suitability Assesment of Materials (SAM). Se utilizó el Índice de Validez de Contenido (IVC) para analizar cada etapa de validez, considerando válidos los iguales o superiores a 0,80 .

Resultados: la cartilla llamada "Cuidados para no caer" se compone de 20 páginas. En la validación de contenido, los profesionales atribuyeron un IVC global de 1,0 para el objetivo, estructura/presentación y relevancia del material. En la evaluación de los pacientes, el IVC global de la cartilla fue de 0,98.

Conclusión: la cartilla educativa es considerada válida respecto al contenido y comprensible por los pacientes, de forma que posee utilización viable en la educación en salud para la prevención de caídas en el hospital.

\section{Autor correspondente}

\section{Maria Aline Moreira Ximenes}

https://orcid.org/0000-0002-1674-3357

E-mail: aline.ximenes11@hotmail.com

\section{DOI}

http://dx.doi.org/10.1590/1982-

0194201900059

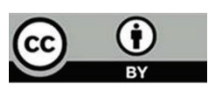

\section{Como citar: \\ Ximenes MA, Fontenele NA, Bastos IB, Macêdo TS, Galindo Neto NM, Caetano JA, et al. Construção e validação de conteúdo de cartilha educativa para prevenção de quedas no hospital. Acta Paul Enferm. 2019;2019;32(4):433-41.}

Universidade Estadual Vale do Acaraú, Sobral, CE, Brasil.

Innstituto Federal de Educação, Ciência e Tecnologia de Pernambuco, Pesqueira, PE, Brasil.

3Universidade Federal do Ceará, Fortaleza, CE, Brasil

Conflitos de interesse: Afirmamos que não há qualquer potencial conflito de interesse políticos e/ou financeiros associados a patentes ou propriedade, provisão de materiais e/ou insumos e equipamentos neste estudo. 


\section{Introdução}

A queda é definida como evento em que o indivíduo sofre impacto no solo, no chão ou em outro nível inferior, a qual constitui problema de saúde pública e pode gerar graves lesôes fatais ou não. Estima-se que 646 mil quedas fatais ocorram, a cada ano, o que a configura como a segunda principal causa de morte por lesão não intencional. Mais de $80 \%$ das mortes relacionadas a quedas ocorrem em países de baixa e média renda. ${ }^{(1)}$

No âmbito hospitalar, indivíduos que sofrem quedas possuem, em média, 12,3 dias a mais de internação e a ocorrência de tal evento adverso pode aumentar em até $61 \%$ os custos hospitalares. ${ }^{(2)}$ Logo, os profissionais de saúde, com destaque para enfermagem, precisam realizar intervençóes direcionadas ao enfrentamento das quedas em hospitais. Dentre tais intervençóes, temos a educação em saúde como estratégia para prevenção de sua ocorrência. (3)

A efetividade de intervençóes educativas na prevenção de quedas é observada em estudos randomizados, realizados na Austrália com 3606 pacientes adultos e com 221 idosos, cujos resultados evidenciam que programas individualizados de educação do paciente combinados com treinamento acerca de cuidados básicos de prevenção são capazes de contribuir para redução das taxas de quedas. ${ }^{(4,5)}$

Durante o processo de educação em saúde, os profissionais podem utilizar materiais educativos impressos que, a partir da organização das informações e da presença de ilustraçóes, favorecem a compreensão das orientaçôes. ${ }^{(6)}$ Dentre estes materiais impressos, destaca-se a cartilha como instrumento útil para descrição de assuntos relacionados à saúde e como recurso de viável utilização diante do seu baixo custo e praticidade na aplicação em âmbito hospitalar. ${ }^{(7)}$

Uma vez que a enfermagem constitui a categoria profissional mais numerosa atuante no ambiente hospitalar, estando em maior tempo de contato com o paciente e que possui a educação em saúde como inerente ao seu exercício profissional, destacase que a disponibilização de cartilha educativa acerca da prevenção de quedas em hospitais, construída e validada por meio de método científico, poderá contribuir para a efetividade do papel educador do enfermeiro acerca da temática.

Vale ressaltar que a disponibilização da cartilha pode favorecer melhorias na assistência direcionada ao paciente hospitalizado ao facilitar a compreensão acerca das condutas de autocuidado para prevenção de eventos adversos como a queda. Ademais, possibilita a realização de futuros estudos que comparem a efetividade de recursos educativos no conhecimento dos pacientes e na prevenção de quedas em hospitais.

Assim, este estudo teve como objetivo construir e validar cartilha educativa para prevenção de quedas no ambiente hospitalar.

\section{Métodos}

Trata-se de estudo metodológico, desenvolvido no período de fevereiro a setembro de 2018, mediante construção e validação de cartilha educativa para prevenção de quedas no ambiente hospitalar.

Para construção da cartilha foi estabelecido as seguintes etapas: 1 - revisão integrativa sobre a temática; 2 - elaboração de storyboard junto ao designer gráfico para auxiliar no processo de desenvolvimento da tecnologia educativa; 3 - validação do material construído pelos profissionais de saúde e avaliação da cartilha pelo público-alvo.

\section{Levantamento bibliográfico}

A coleta de dados foi realizada em abril de 2018, para a seleção dos artigos sobre quedas no ambiente hospitalar, foram consultadas as bases de dados PubMed, LILACS, MEDLINE, BDEnf, Scielo, SCOPUS e CINAHL. Os descritores utilizados foram: "Enfermagem/Nursing"; "Acidentes por Quedas/Accidental Falls", "Hospitalização/ Hospitalization", "Segurança do Paciente/Patient Safety". A estratégia de busca utilizada foi: "Enfermagem/Nursing" AND "Acidentes por Quedas/Accidental Falls" AND "Hospitalização/ Hospitalization" AND "Segurança do Paciente/ Patient Safety”. Os critérios de inclusão foram: artigos disponibilizados na íntegra nos idiomas inglês, espanhol e português e publicados a partir de 2008. 
Foram excluídos editoriais, estudos reflexivos, manuscritos repetidos e que não abordavam a temática do estudo.

Foram encontradas 98 publicações e, após a leitura dos resumos e artigos na íntegra, foram selecionados 16 artigos para leitura reflexiva e síntese dos pontos mais relevantes, os quais nortearam a elaboração do conteúdo da cartilha educativa. Foram coletadas informaçóes referentes ao título, ano de publicação, país, idioma, objetivo, método, resultados, conclusão e nível de evidência.

Após a compilação dos dados obtidos com os artigos selecionados na revisão integrativa, também foram incluídas informaçôes presentes nos protocolos de prevenção de quedas disponibilizados pelo Ministério da Saúde, Agência Nacional de Vigilância Sanitária (ANVISA) e Organização Mundial de Saúde. ${ }^{(8,9)}$

\section{Construção do material educativo}

Após elencar o conteúdo pertinente para a cartilha, foi elaborado roteiro com informaçôes, cenários e textos que deveriam estar presentes no material. Posteriormente houve adequação da linguagem científica, tornando-a mais apropriada ao público, independente do grau de instrução.

Em seguida, o material foi entregue a profissional especialista na área de designer para desenvolver as ilustraçôes e realizar a diagramação do material. A escolha pelo profissional é justificada pela experiência em desenvolver materiais educativos para o Programa de Pós-graduação em Enfermagem da Universidade Federal do Ceará, contribuindo com a construção de tecnologias relacionadas à dissertaçôes e teses.

A diagramação e estrutura do texto/frases foram realizadas, pelo designer, mediante acompanhamento do pesquisador e conforme recomendaçóes para textos de tecnologias educativas ${ }^{(10)}$ e as ilustraçóes coloridas foram criadas no programa Corel Draw X7.

\section{Validação de conteúdo com juízes}

Após construída, a validação da cartilha foi realizada por enfermeiros com expertise em assistência no ambiente hospitalar, segurança do paciente ou tecnologias educacionais. $\mathrm{O}$ tamanho da amostra foi definido a partir da fórmula $\mathrm{n}=\mathrm{Za}^{2} \cdot \mathrm{P}(1-\mathrm{P}) / \mathrm{e}^{2}$. Os valores estipulados foram $\mathrm{Za}$ (nível de confiança) = 95\%, P (proporção de concordância dos juízes) = $85 \%$, e (diferença aceita do que se espera) $=15 \%$, o que resultou em 22 juízes. ${ }^{(11)}$

A busca pelos juízes ocorreu entre docentes dos cursos de Enfermagem das universidades públicas do estado do Ceará com expertise em segurança do paciente, tecnologias educacionais e enfermagem. Foi solicitado também, através da amostragem de bola de neve, a indicação de outros docentes com perfil para participar da validação. A partir da indicação, foi avaliado o currículo lattes para verificar se os profissionais indicados atendiam aos critérios de inclusão estabelecidos.

$\mathrm{Na}$ seleção dos profissionais, foram utilizados os critérios de Jasper ${ }^{(12)}$ como possuir conhecimento/ habilidade na temática (orientação de dissertaçôes e teses relativas à segurança do paciente ou tecnologias educacionais); possuir conhecimento/habilidade através da experiência profissional (ter experiência docente na área de enfermagem clínica ou ter experiência assistencial no ambiente hospitalar) e possuir expertise em determinado tipo de estudo (experiência no desenvolvimento de tecnologias educacionais impressas, participação de bancas avaliadoras relacionadas à temática e ter artigos publicados sobre segurança do paciente ou tecnologias educacionais). Assim, foram convidados 45 profissionais, dos quais 23 não retornaram o contato, o que resultou em amostra de 22 juízes.

Para coletar os dados, foi construído formulário pela ferramenta do Google Forms que foi compartilhado via e-mail, junto com o Termo de Consentimento Livre e Esclarecido (TCLE). Foi estipulado prazo de 14 dias para avaliação da cartilha e preenchimento do instrumento.

No formulário, foram inseridas as imagens da cartilha juntamente com os 18 itens do instrumento de validação, que continha variáveis acerca do objetivo (propósitos, metas ou finalidades da cartilha), estrutura/apresentação (organização, estrutura, linguagem, coerência e tamanho do texto) e relevância do material (significância, impacto, motivação e interesse para leitura da cartilha). ${ }^{(13)}$ Cada um desses tópicos presentes no instrumento conti- 
nha frases afirmativas sobre os itens e, após a leitura do material, os juízes poderiam avaliar o item como adequado, parcialmente adequado ou inadequado.

Foi incluído também espaço para sugestóes a serem realizadas pelos juízes. Todas as sugestóes enviadas pelos juízes foram organizadas e analisadas conforme as variáveis do instrumento (objetivo, estrutura/apresentação e relevância do material), as quais foram devidamente acatadas. Foi solicitado reformulação de algumas frases para facilitar a compreensão do público-alvo, bem como a melhoria de ilustrações como ressaltar cores vibrantes e incluir imagens representativas das práticas recomendadas no texto.

\section{Avaliação pelo público-alvo}

Após a validação de conteúdo, o material educativo foi avaliado por 22 pacientes hospitalizados em clínica médica de hospital público em Sobral, Ceará, Brasil. A escolha deste cenário é justificada pelo fato de que a cartilha aborda a prevenção de quedas em pacientes internados na enfermaria hospitalar e o setor escolhido ter semelhança com as ilustraçôes presentes no material educativo. Para a inclusão na amostra, adotou-se os seguintes critérios de inclusão: ser alfabetizado e estar clinicamente estável durante a leitura do material. Foram excluídos pacientes com alteraçóes visuais e cognitivas.

Aplicou-se o instrumento Suitability Assesment of Materials (SAM) traduzido e validado para o português brasileiro que contém 30 itens que contempla a avaliação de objetivos, organização, linguagem, aparência e motivação do material impresso.

(14) Foi acrescentado um tópico que continha dados clínico-epidemiológicos do paciente e outro tópico em que o paciente classificava o material em aprovado, aprovado com modificaçóes, reprovado com qualidade e reprovado. Além disso, também foi solicitado que os mesmos indicassem sugestóes para melhorar a compreensão ou ilustração do material.

A seleção dos participantes ocorreu a partir da identificação dos pacientes internados e da aplicação dos critérios de inclusão e exclusão. Após a explicação do objetivo do estudo, os pacientes que aceitaram participar, assinaram o TCLE. Em seguida foi entregue a cartilha e o instrumento de coleta de dados. Cada participante foi instruído a realizar a leitura da cartilha, analisar o texto e as imagens e, em seguida, responder ao formulário.

\section{Análise dos dados}

Foi realizada análise descritiva dos dados referente à caracterização dos juízes e pacientes. Para verificação da validade de conteúdo da cartilha, foi utilizado o Índice de Validade de Conteúdo (IVC): foi calculado o I-CVI (Item-level Content Validity Index) referente a cada item do instrumento e o IVC global. A cartilha foi considerada válida quando o item obteve IVC igual ou superior a 0,8 . Ademais, foi utilizado o teste binomial no software $\mathrm{R}$ para verificar a concordância, estatisticamente, igual ou superior a 0,80 dos juízes e pacientes, separadamente. O nível de significância adotado no estudo foi de 5\%.

\section{Aspectos éticos}

O projeto foi aprovado pelo Comitê de Ética em Pesquisa da Universidade Estadual Vale do Acaraú, sob o parecer no 2.933.103, conforme a resolução $466 / 2012$.

\section{Resultados}

A cartilha para prevenção de quedas intitulada "Cuidado para não cair nessa" foi construída com 20 páginas, composta por capa, contracapa, ficha técnica, folha de rosto e página de apresentação. Os personagens presentes no material educativo foram uma enfermeira denominada Ana, uma paciente, Maria, um acompanhante, José, e um mascote, chamado Alertinha, que interage com o leitor durante todo o conteúdo exposto.

O conteúdo foi dividido nos seguintes tópicos: "Situaçóes de risco" (apresenta a definição e caracterização de quedas); "Fatores associados a quedas" (contempla os riscos extrínsecos e intrínsecos); "Como evitar complicações" (aborda medidas de prevenção para o paciente e acompanhante); "Ambiente seguro" (possui dicas para manter o ambiente da enfermaria mais seguro); "Exercícios fazem bem" (orientaçóes de exercícios para fortalecimento da musculatura); "Super equipe" (apresen- 
ta atribuiçóes da equipe de enfermagem, voltadas à prevenção de quedas, e as condutas diante de uma situação de queda); "Jogo de sete erros" (lista fatores de risco presentes no ambiente hospitalar).

Para tornar a cartilha didática e compreensível, a abordagem do tema ocorreu de forma clara e objetiva, com títulos e imagens destacadas, linguagem simples, sequência lógica das informações, além de balóes com texto para ratificar o conteúdo. Assim, as informaçóes foram expressas a partir de diálogos entre os personagens em que a enfermeira interage com paciente e acompanhante, apresentando orientações para prevenção de quedas. Vale ressaltar que o cenário ilustrado correspondia à enfermaria clínica hospitalar. São apresentadas (Figura 1) algumas páginas da versão final da cartilha.

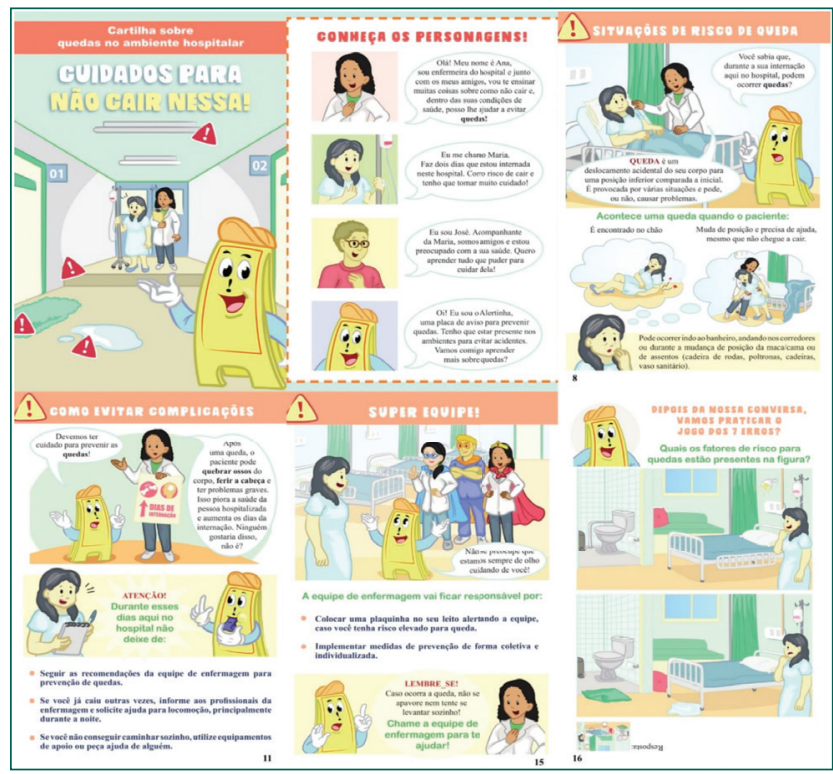

Figura 1. Páginas da Cartilha sobre quedas do ambiente hospitalar "Cuidados para não cair nessa!"

Na validação de conteúdo, realizada por 22 enfermeiros, 11 eram mestres, seis doutores e cinco possuíam especialização. Em relação à área de atuação, 12 atuavam na docência, dois na pesquisa, um na gestão e sete na assistência. No que se refere a experiência docente, 14 possuíam experiência na temática e 16 já haviam participado de treinamentos ou cursos sobre quedas. Os itens avaliados e os respectivos valores dos IVCs, referentes aos objetivos, estrutura/apresentação e relevância, se encontram detalhados na tabela 1.
Tabela 1. Concordância dos profissionais quanto aos itens da cartilha

\begin{tabular}{|c|c|c|c|}
\hline Variáveis & $\mathrm{n}(\%)^{\star}$ & I-CVI & p-valort \\
\hline \multicolumn{4}{|l|}{ 1. Objetivos } \\
\hline 1.2 Contempla tema proposto & $22(100)$ & 1 & 1 \\
\hline $\begin{array}{l}\text { 1.3 As informações/conteúdos são adequadas ao } \\
\text { processo e ensino-aprendizagem }\end{array}$ & $22(100)$ & 1 & 1 \\
\hline 1.4 Esclarece possíveis dúvidas sobre o tema abordado & $22(100)$ & 1 & 1 \\
\hline 1.5 Proporciona reflexão sobre o tema & $22(100)$ & 1 & 1 \\
\hline 1.6 Incentiva mudança de comportamento & $22(100)$ & 1 & 1 \\
\hline \multicolumn{4}{|l|}{ 2. Estrutura e Apresentação } \\
\hline $\begin{array}{l}\text { 2.1 Mensagens estão apresentadas em linguagem } \\
\text { adequada ao público-alvo }\end{array}$ & $22(100)$ & 1 & 1 \\
\hline 2.2 Linguagem apropriada ao material educativo & $22(100)$ & 1 & 1 \\
\hline $\begin{array}{l}2.3 \text { Linguagem interativa, permitindo envolvimento ativo } \\
\text { no processo educativo }\end{array}$ & $22(100)$ & 1 & 1 \\
\hline 2.4 As informações estão corretas & $22(100)$ & 1 & 1 \\
\hline 2.5 As informações são objetivas & $22(100)$ & 1 & 1 \\
\hline 2.6 As informações são esclarecedoras & $22(100)$ & 1 & 1 \\
\hline 2.7 As informações são necessárias & $22(100)$ & 1 & 1 \\
\hline 2.8 As ideias estão em sequência lógica & $22(100)$ & 1 & 1 \\
\hline 2.90 tema é atual & $22(100)$ & 1 & 1 \\
\hline 2.100 tamanho do texto é adequado & $22(100)$ & 1 & 1 \\
\hline \multicolumn{4}{|l|}{ 3. Relevância } \\
\hline 3.1 A cartilha estimula 0 aprendizado & $22(100)$ & 1 & 1 \\
\hline 3.2 Contribui para o conhecimento na área & $22(100)$ & 1 & 1 \\
\hline 3.3 Desperta interesse pelo tema & $22(100)$ & 1 & 1 \\
\hline
\end{tabular}

*Percentual de concordância no item; I-CVI = Item-Level Content Validity Index; †Teste binomial

Conforme observado na tabela 1 , os 18 itens foram avaliados pelos juízes como adequados, houve nível de concordância de $100 \%$, a concordância acerca dos objetivos do material educativo foi unânime, assim como seu conteúdo e relevância para a prática clínica. O IVC global na validação com juízes foi igual a 1,0 que é considerado padrão-ouro. Diante desse resultado, foi realizada apenas uma rodada na validação com os juízes tendo em vista que as sugestóes para modificaçóes foram mínimas e não invalidariam o material já avaliado e validado. Dentre as sugestóes feitas pelos juízes, destacam-se as principais: explicitar no título a prevenção de quedas no ambiente hospitalar, realçar as cores da capa, rever alguns termos de difícil compreensão e acrescentar figuras que ilustrem os exemplos de exercícios para fortalecer a musculatura. Todas essas modificações foram acatadas e foi solicitado que o designer realizasse tais alteraçóes. A versão final da cartilha foi avaliada pelo público-alvo representado por pacientes internados que, em sua maioria, eram homens com idade média de 28,7 anos, solteiros, pardos e com ensino médio completo. O tempo médio de internação foi de 11,6 dias devido acidentes automobilísticos, pós-operatório e compli- 
cações como infecçóes. A maioria dos entrevistados possuía histórico de quedas tanto domésticas como hospitalares. A concordância dos pacientes na avaliação da cartilha é apresentada na tabela 2 .

Tabela 2. Concordância dos pacientes internados em clínica médica, quanto aos itens da cartilha

\begin{tabular}{|c|c|c|c|}
\hline Variáveis & $n(\%)^{*}$ & I-CVI & p-valor† \\
\hline \multicolumn{4}{|l|}{ 1. Objetivos } \\
\hline $\begin{array}{l}\text { 1.1 Atende aos objetivos dos pacientes internados e os } \\
\text { ajuda a compreender os riscos de quedas. }\end{array}$ & $21(95,4)$ & 0,95 & 0,972 \\
\hline $\begin{array}{l}1.2 \text { É capaz de promover reflexão sobre os cuidados } \\
\text { necessários na prevenção de quedas. }\end{array}$ & $22(100)$ & 1 & 1 \\
\hline $\begin{array}{l}\text { 1.3 Está adequada para ser usada por qualquer profissional } \\
\text { da área da saúde. }\end{array}$ & $22(100)$ & 1 & 1 \\
\hline \multicolumn{4}{|l|}{ 2.0rganização } \\
\hline 2.1 A capa da cartilha está atraente. & $22(100)$ & 1 & 1 \\
\hline $\begin{array}{l}\text { 2.2 A capa da cartilha contempla as informações do } \\
\text { material. }\end{array}$ & $22(100)$ & 1 & 1 \\
\hline $\begin{array}{l}2.30 \text { tamanho do título e dos conteúdos nos tópicos está } \\
\text { adequado. }\end{array}$ & $22(100)$ & 1 & 1 \\
\hline 2.4 Os temas retratam aspectos-chave importantes. & $20(90,9)$ & 0,90 & 0,863 \\
\hline $\begin{array}{l}2.5 \text { Há coerência entre as informações da capa, } \\
\text { apresentação, sumário e conteúdo da cartilha. }\end{array}$ & $22(100)$ & 1 & 1 \\
\hline 2.60 papel do material impresso está apropriado. & $22(100)$ & 1 & 1 \\
\hline 2.70 número de páginas está adequado. & $20(90,9)$ & 0,90 & 0,863 \\
\hline $\begin{array}{l}2.80 \text { material tem tamanho adequado, ou seja, não é } \\
\text { extenso nem cansativo. }\end{array}$ & $21(95,4)$ & 0,95 & 0,972 \\
\hline \multicolumn{4}{|l|}{ 3. Linguagem } \\
\hline 3.1 A redação está em estilo adequado. & $22(100)$ & 1 & 1 \\
\hline 3.20 texto é vívido e interessante. & $22(100)$ & 1 & 1 \\
\hline $\begin{array}{l}3.30 \text { vocabulário é acessivel com palavras simples e } \\
\text { comuns. }\end{array}$ & $22(100)$ & 1 & 1 \\
\hline $\begin{array}{l}3.4 \text { Todos os conceitos importantes são abordados de } \\
\text { forma clara e objetiva. }\end{array}$ & $22(100)$ & 1 & 1 \\
\hline $\begin{array}{l}3.5 \text { Há associação entre o tema de cada sessão e o texto } \\
\text { correspondente. }\end{array}$ & $22(100)$ & 1 & 1 \\
\hline $\begin{array}{l}3.60 \text { texto apresenta interação com orientações entre } \\
\text { profissional e público-alvo. }\end{array}$ & $22(100)$ & 1 & 1 \\
\hline $\begin{array}{l}3.70 \text { texto é escrito de forma que o público-alvo é o centro } \\
\text { das atenções, ou seja, o paciente é mais importante. }\end{array}$ & $21(95,4)$ & 0,95 & 0,972 \\
\hline \multicolumn{4}{|l|}{ 4. Aparência } \\
\hline 4.1 A composição visual é atrativa. & $22(100)$ & 1 & 1 \\
\hline 4.2 As páginas ou sessões parecem organizadas. & $22(100)$ & 1 & 1 \\
\hline 4.30 número de figuras é suficiente. & $21(95,4)$ & 0,95 & 0,972 \\
\hline 4.4 As figuras são apresentadas em tamanho adequado. & $22(100)$ & 1 & 1 \\
\hline $\begin{array}{l}\text { 4.5 As figuras são simples, apropriadas e de tracejado de } \\
\text { fácil compreensão. }\end{array}$ & $22(100)$ & 1 & 1 \\
\hline 4.6 As figuras são autoexplicativas. & $21(95,4)$ & 0,95 & 0,972 \\
\hline 4.7 As figuras são familiares para os leitores. & $22(100)$ & 1 & 1 \\
\hline 4.8 As figuras estão integradas ao texto (bem localizadas). & $22(100)$ & 1 & 1 \\
\hline \multicolumn{4}{|l|}{ 5. Motivação } \\
\hline 5.1 A cartilha é apropriada para sua idade, sexo e cultura. & $21(95,4)$ & 0,95 & 0,972 \\
\hline $\begin{array}{l}5.2 \text { A cartilha apresenta sequência lógica sobre os } \\
\text { cuidados para prevenir quedas. }\end{array}$ & $22(100)$ & 1 & 1 \\
\hline 5.3 A cartilha desperta interesse e curiosidade. & $22(100)$ & 1 & 1 \\
\hline $\begin{array}{l}5.4 \text { A cartilha promove mudança de comportamento e } \\
\text { atitude. }\end{array}$ & $22(100)$ & 1 & 1 \\
\hline 5.50 conteúdo da cartilha mantém a dinâmica da leitura. & $22(100)$ & 1 & 1 \\
\hline 5.60 contéudo da cartilha motiva a leitura até o final. & $21(95,4)$ & 0,95 & 0,972 \\
\hline 5.70 uso da cartilha se faz relevante. & $22(100)$ & 1 & 1 \\
\hline
\end{tabular}

*Percentual de concordância no item; I-CVI = Item-Level Content Validity Index; †Teste binomial
Dos 33 itens avaliados pelos pacientes, 24 apontaram 100\% de concordância (IVC igual a 1,0). Outros sete obtiveram IVC de 0,95 e dois IVC igual a 0,90 . O IVC global da cartilha na validação com pacientes foi de 0,98. Os ajustes sugeridos pelos pacientes foram referentes ao aumento no tamanho da fonte e à troca do tipo de papel utilizado para impressão. As sugestôes foram acatadas para versão final da cartilha.

\section{Discussão}

A divulgação de materiais educativos impressos é eficaz para contribuir com melhorias na área da saúde, conforme verificado nos resultados de pesquisa longitudinal realizada durante quatro anos, no sul do Brasil, que evidenciou associação entre a distribuiçãao de folders/cartazes e a diminuição do índice de quedas. ${ }^{15)}$ Torna-se, então, pertinente o desenvolvimento de estudos metodológicos que contemplem a construção e validação de materiais educativos em saúde para disponibilização nos serviços de saúde.

Os conteúdos abordados na cartilha contribuem para divulgação de informaçôes importantes preconizadas pelo protocolo de prevenção de quedas, integrante do Programa Nacional de Segurança do Paciente, do Ministério da Saúde. ${ }^{(8)}$ Assim, a partir da diagramação do conteúdo em caixas de textos e tópicos e com ilustraçóes do texto, o material fica mais fácil para ser compreendido durante a leitura.

Tal fato converge com a proposta de outros materiais educativos para prevenção de quedas, construídos por organizaçóes de gerenciamento e prevenção de quedas na Irlanda e hospitais na Irlanda do Norte e Austrália. Estes materiais, impressos em forma de livreto, tiveram como público-alvo idosos e abordaram as principais situaçóes de risco, além de informaçóes relacionadas à prevenção de quedas. ${ }^{(16-18)}$ Observa-se, assim, a preocupação internacional para disseminar informaçōes em tecnologias impressas que contribuam para educação em saúde acerca da temática.

Durante a etapa de validação pelos profissionais, o IVC foi igual a 1,0 em todos os tópicos ana- 
lisados. Tal achado corrobora com outros estudos metodológicos realizados no Brasil que obtiveram resultados satisfatórios na validação de conteúdo de cartilha para crianças com hidrocefalia e seus cuidadores $^{(19,20)}$ que obteve IVC entre 0,9 e 1,0 e cartilha para prevenção de síndrome metabólica em adolescentes ${ }^{(21)}$ que obteve IVC de 1,0 na maioria dos itens analisados. $\mathrm{O}$ processo de validação com avaliação de itens referentes ao objetivo, estrutura/apresentação e relevância é importante para que materiais educativos não possuam informaçóes equivocadas ou incompletas que possam induzir a população-alvo ao erro ou dificultar o entendimento da temática.

Além de possuir informaçóes corretas e serem válidos quanto ao conteúdo, é necessário que os materiais educativos em saúde sejam compreensíveis pelo público-alvo. Nessa perspectiva, os profissionais que realizaram a validação de conteúdo sugeriram pequenos ajustes relacionados a adequação de linguagem da cartilha em frases que poderiam dificultar a interpretação dos pacientes.

Tal situação assemelha-se com achado de estudos metodológicos brasileiros sobre validação de livreto para prevenção de transmissão vertical de HIV e para pacientes com câncer de cabeça e pescoço submetidos à radioterapia que também evidenciaram a necessidade de modificação da linguagem de forma a deixar mais claro o conteúdo aos leitores, conforme sugestôes dos juízes. ${ }^{(22,23)}$ Assim, observase que sugestôes de ajustes no texto de tecnologias educativas são recorrentes e possuem relevância para que o material possa ser compreendido pelo maior número possível de pessoas.

Após a realização das alteraçôes propostas, foi possível constatar que os pacientes compreenderam facilmente as informaçôes contidas no material impresso tendo em vista que todos os tópicos relacionados a linguagem apresentaram IVC igual a 1,0, o que indica a clareza da escrita. Esses achados convergem com estudo sobre materiais educativos impressos no Texas, que tratava de segurança no uso de medicamentos com 300 pacientes, os resultados mostraram que a ferramenta de educação em saúde era de fácil compreensão e linguagem acessível aos pacientes. ${ }^{(24)}$
Dessa forma, os materiais educativos, uma vez compreensíveis e atrativos, caracterizam-se como alternativa de sensibilização do paciente de forma que este possa ser ativo no seu autocuidado. Acerca disso, no presente estudo, foi possível comprovar a viabilidade para utilização da cartilha, pois os pacientes manifestaram interesse em ler o material e aprender sobre prevenção de quedas.

No Reino Unido, estudo sobre utilização de livreto para prevenção de quedas com idosos na comunidade também demonstrou boa aceitabilidade pelos pacientes e aplicabilidade prática do material. (25) Assim, é importante que estudos que envolvem novas tecnologias educacionais avaliem sua aplicabilidade no contexto em que serão utilizadas. ${ }^{(26)}$

Vale ressaltar que o manual educativo não foi construído com intuito de substituir as orientaçôes verbais fornecidas pelo enfermeiro durante assistência de enfermagem, mas sim para reforçar as orientaçôes e solucionar dúvidas, pois o paciente poderá consultar o material sempre que tiver interesse. ${ }^{(25)}$ Sugere-se, então, que os enfermeiros utilizem a cartilha durante as estratégias educativas e que, nesse momento, essa tecnologia facilite a construção de conhecimentos entre enfermeiro-paciente-familiar, além de favorecer o esclarecimento de dúvidas.

A prática de construção, validação e aplicação de materiais educacionais do tipo cartilha tem apresentado resultados positivos em estudos nacionais e internacionais. Logo, é importante que instrumentos de orientação ao paciente sobre quedas sejam desenvolvidos e validados para utilização na prática clínica com intuito de contribuir na cultura de segurança do paciente no contexto hospitalar. Ressalta-se que uma das prioridades na redução de ocorrência de quedas em pacientes hospitalizados é o cuidado multiprofissional com açôes que promovam a educação do paciente e familiares. ${ }^{\left({ }^{(8)}\right.}$

Assim, o presente estudo contribui para o avanço científico na ciência da enfermagem ao disponibilizar, para o meio assistencial e acadêmico, cartilha com enfoque educativo que foi validada por juízes e avaliada pelo público-alvo (pacientes), podendo ser utilizada na prática clínica

Acredita-se que é pertinente, após a construção e validação, a verificação da efetividade do 
material educativo na orientação do paciente em relação à prevenção de quedas no ambiente hospitalar. Este momento poderá ser realizado de modo individual ou coletivo com pacientes e acompanhantes.

Este estudo apresenta limitaçôes referentes à generalizaçáo dos resultados tendo em vista que a cartilha aborda prevenção de quedas no contexto hospitalar, o que dificultou a discussão dos achados com estudos com a mesma temática. Outra limitação é que o material foi avaliado apenas por pacientes usuários do Sistema Único de Saúde, no setor de clínica médica, de forma que os resultados obtidos podem divergir da realidade de pacientes atendidos na rede privada de assistência à saúde ou internados em outros setores.

\section{Conclusão}

A cartilha educativa "Cuidado para náo cair nessa" foi construída a partir de revisão da literatura científica e da literatura de referência na temática (protocolos de prevenção de quedas disponibilizados pelo Ministério da Saúde, ANVISA e Organização Mundial de Saúde). Na validação de conteúdo, foi considerada válida por juízes (enfermeiros), em relação aos objetivos, estrutura/apresentação e relevância $(\mathrm{IVC}=1,0)$ e avaliada como compreensível por pacientes internados em clínica médica (IVC=0,98). É apropriado que durante a avaliaçáo de materiais educativos, o público-alvo possa opinar quanto ao conteúdo, linguagem e aparência para que o material se torne adequado para a população a qual se destina. Consequentemente, o material alcançará o seu objetivo quando for aplicado durante a prática clínica. Dessa forma, recomenda-se que em todo estudo metodológico seja valorizado a etapa de avaliação pelo público-alvo. Disponibiliza-se material educativo viável para ser utilizado no contexto hospitalar, que pode favorecer a prevenção de quedas ao possibilitar o acesso dos pacientes ao conhecimento de cuidados importantes. Assim, este material vem a contribuir com a segurança do paciente bem como a melhoria da qualidade da assistência e educação em saúde realizada pela enfermagem.

\section{Agradecimentos}

A Santa Casa de Misericórdia de Sobral-CE pelo financiamento dessa pesquisa (Edital DEPE 02/2017).

\section{Colaborações}

Ximenes MAM contribuiu na concepção do projeto, coleta e interpretação dos dados e redação do artigo. Fontenele NAO, Bastos IB, Macêdo TS contribuíram na coleta e interpretação dos dados. Galindo Neto NM, Caetano JÁ e Barros LM, contribuíram com a redação do artigo, revisão crítica do conteúdo do artigo e aprovação da versão final a ser publicada.

\section{Referências}

1. World Health Organization (WHO). Falls [Internet]. Genève: WHO; c2019. [cited 2018 Nov 15]. Available from: http://www.who.int/newsroom/fact-sheets/detail/falls

2. Avanecean D, Calliste D, Contreras T, Lim Y, Fitzpatrick A. Effectiveness of patient-centered interventions on falls in the acute care setting compared to usual care: a systematic review. JBI Database System Rev Implement Rep. 2017;15(12):3006-48.

3. Bittencourt VLL, Graube SL, Stumm EMF, Battisti IDE, Loro MM, Winkelmann ER. Factors associated with the risk of falls in hospitalized adult patients. Rev Esc Enferm USP. 2017;51:e03237.

4. Hill AM, McPhail SM, Waldron N, Etherton-Beer C, Ingram K, Flicker $L$, et al. Fall rates in hospital rehabilitation units after individualised patient and staff education programmes: a pragmatic, stepped-wedge, cluster-randomised controlled trial. Lancet. 2015;385(9987):2592-9.

5. Mikolaizak AS, Lord SR, Tiedemann A, Simpson P, Caplan GA, Bendall $J$, et al. A multidisciplinary intervention to prevent subsequent falls and health service use following fall-related paramedic care: a randomised controlled trial. Age Ageing. 2017; 46 (2):200-7.

6. Grudniewicz A, Bhattacharyya 0, McKibbon KA, Straus SE. Redesigning printed educational materials for primary care physicians: design improvements increase usability. Implementation Sci. 2015;10:156.

7. Siddharthan T, Rabin T, Canavan ME, Nassali F, Kirchhoff P, Kalyesubula $\mathrm{R}$ et al. Implementation of patient-centered education for chronicdisease management in Uganda: an effectiveness study. PLoS One. 2016;11(11):e0166411.

8. Brasil. Ministério da Saúde. Protocolo prevenção de quedas de 03 de maio de 2013. [Internet]. Brasília (DF); 2013 [citado 2018 Nov 16]. Disponível em: http://www.saude.mt.gov.br/upload/controleinfeccoes/ pasta12/protocolos_cp_n6_2013_prevencao.pdf

9. World Health Organization (WHO). WHO Global Report on Falls Prevention in Older Age. [Internet]. Genève: WHO; 2007. [cited 2018 
Nov 16]. Available from: https://extranet.who.int/agefriendlyworld/wpcontent/uploads/2014/06/WHoGlobal-report-on-falls-prevention-inolder-age.pdf

10. Deatrick D, Aalberg J, Cawley J. A guide to creating and evaluating patient materials. guidelines for effective print communication [Internet]. Portland: MaineHealth; 2010. [cited 2018 Nov 16]. Available from: https://mainehealth.org/-/media/community-education-program-cep/ health-literacy/mh-print-guidelines.pdf?la=en

11. Lopes MVO, Silva VM, Araujo TL. Methods for establishing the accuracy of clinical indicators in predicting nursing diagnoses. Int J Nurs Knowl. 2012;23(3):134-9.

12. Jasper MA. Expert: a discussion of the implications of the concept as used in nursing. J Adv Nurs. 1994; 20(4):769-76.

13. Leite SS, Áfio AC, Carvalho LV, Silva JM, Almeida PC, Pagliuca LM. Construção e validação de instrumento de validação de conteúdo educativo em saúde. Rev Bras Enferm. 2018; 71(4): 1635-41.

14. Sousa CS, Turrini RN. Validação de constructo de tecnologia educativa para pacientes mediante aplicação da técnica Delphi. Acta Paul Enferm. 2012;25(6):990-6.

15. Luzia MF, Cassola TP, Suzuki LM, Dias VL, Pinho LB, Lucena AF. Incidence of falls and preventive actions in a University Hospital. Rev Esc Enferm USP. 2018;52:e03308.

16. Don't fall for it, falls can be prevented! A guide to preventing falls for older people. [Internet]. Government of South Australia. 2011. [cited 2018 Nov 15]. Available from:https://www.health.gov.au/internet/main/publishing. nsf/Content/E23F5F7BF8F07264CA257BF0002043F5/\$File/Don't\%20 fall\%20for\%20it.pdf

17. Falls prevention. Working together to prevent falls [Internet]. Lisburn: Health Development Departament. [cited 2018 Nov 15]. Available from: http://www.setrust.hscni.net/pdf/Falls_advice_booklet.pdf
18. Falls Prevention Educational Information [Internet]. UHW Falls Management Group; 2014. [cited 2018 Nov 15]. Available from: https://www.hse.ie/eng/health/hl/selfmanagement/donegal/ programmesservices/falls-prevention/falls-prevention-educationbooklet.pdf

20. Tavares PA, Filho PT, Ferreira AS, Avila MA. Construction and validation of educational material for children with hydrocephalus and their informal caregivers. World Neurosurg. 2018; 114:381-90.

21. Moura $\mathbb{H}$, Silva AF, Rocha AE, Lima LH, Moreira TM, Silva AR. Construção e validação de material educativo para prevenção de síndrome metabólica em adolescentes. Rev Lat Am Enfermagem. 2017:25:e2934.

22. Lima AC, Bezerra KC, Sousa DM, Rocha JF, Oriá MO. Construção e Validação de cartilha para prevenção da transmissão vertical do HIV. Acta Paul Enferm. 2017; 30(2):181-9.

23. Cruz FO, Ferreira EB, Vasques $\mathrm{Cl}$, Mata LR, Reis PE. Validation of an educative manual for patients with head and neck cancer submitted to radiation therapy. Rev Lat Am Enfermagem. 2016;24:e2706.

24. Cruz M, Reddy A, Balankari V, Epner M, Hume SF, Wu J, et al. The impact of an educational program on patient practices for safe use, storage, and disposal of opioids at a Comprehensive Cancer Center. Oncologist. 2017; 22(1):115-21.

25. Cockayne S, Rodgers S, Green L, Fairhurst C, Adamson J, Scantlebury A, et al. Clinical effectiveness and cost-effectiveness of a multifaceted podiatry intervention for falls prevention in older people: a multicentre cohort randomised controlled trial (the REducing Falls with ORthoses and a Multifaceted podiatry intervention trial). Health Technol Assess. 2017; 21(24):1-198

26. Galindo Neto NM, Caetano JA, Barros LM, Silva TM, Vasconcelos EM. Primeiros socorros na escola: construção e validação de cartilha educativa para professores. Acta Paul Enferm. 2017; 30(1):87-93. 